\title{
Extubação precoce em cirurgia cardíaca infantil: procedimentos e resultados em seis anos de experiência
}

\author{
José Augusto BÁUCIA*, Miguel BARBERO-MARCIAL*
}

RBCCV 44205-182

BAUCIA, J. A. \& BARBERO-MARCIAL, M. - Extubaçäo precoce em cirurgia cardíaca infantil: procedimentos e resultados em seis anos de experiência. Rev. Bras. Cir. Cardiovasc., 7(3):215-220, 1992.

RESUMO: De 441 crianças portadoras de cardiopatias congênitas submetidas a intervençōes cirúrgicas, $372(84 \%)$ foram extubadas em sala cirúrgica, imediatamente após o procedimento baseado em critérios clínicos, saturaçāo cutânea de $\mathrm{O}_{2}$ e $\mathrm{pCO}_{2}$ no ar expirado. As complicaçōes pós-operatórias nāo apresentaram correlaçāo com o procedimento, observando-se, inclusive, baixo índice de complicaçōes pulmonares. Concluiu-se que a maioria das crianças, incluindo muitas com lesões complexas e hipertensão pulmonar préoperatória, podem ser extubadas precocemente com segurança e diminuiçāo das complicaçōes pulmonares secundárias à ventilação mecânica, bem como reduçāo do período de permanência em UTI, e de hospitalizaçāo, stress da criança, dos familiares e os custos hospitalares.

DESCRITORES: cirurgia cardíaca pediátrica, extubação precoce.

\section{INTRODUÇĀO}

A necessidade e a duração da assistência ventilatória mecânica, no pós-operatório de crianças portadoras de cardiopatias congênitas submetidas a intervençōes cirúrgicas, constitui motivo de controvérsia na literatura. Nos últimos anos, entretanto, vêm-se avolumando relatos de bons resultados com a extubação precoce após intervençōes cardíacas, com diminuição das complicaçōes pulmonares pósoperatórias.

O objetivo deste trabalho é o de avaliar o resultado pós-operatório imediato com a extubação em sala cirúrgica, de crianças portadoras de cardiopatias congênitas submetidas a intervençōes cirúrgicas em nosso Serviço, nos últimos seis anos.

\section{CASUÍSTICA E MÉTODOS}

No período de janeiro de 1987 a agosto de 1992 , foram realizadas 441 cirurgias cardíacas em crianças portadoras de cardiopatias congênitas no Hospital Sírio-Libanês. A idade variou de 1 dia a 16 anos (média de 6 anos) (Tabela 1). Os procedimentos cirúrgicos realizados encontram-se relacionados na Tabela 2. Nenhum paciente foi excluído do estudo, mesmo aqueles com comprometimento da funçăo pulmonar no pré-operatório.

A medicação pré-anestésica empregada foi o Dormonid na dosagem de $0,25 \mathrm{mg} / \mathrm{kg}$, administrado aproximadamente 1 hora antes do procedimento, exceto em neonatos e lactentes. A indução anestésica foi realizada em $90 \%$ dos pacientes por via inalatória com Forane ou Fluotane, e nos casos considerados mais graves e ocasionalmente em crianças maiores, com a Ketamina via intramuscular, na dosagem de 10 $\mathrm{mg} / \mathrm{kg}$. Como agente curarizante preferiu-se o Pavulon ao Quelicin. A manutenção foi quase sempre por via inalatória com Forane, embora ocasionalmente tenha-se empregado o Fentanil intermitentemente em microdoses. Na recuperação pós-anestésica proce-

Trabalho realizado no Hospital Sírio Libanês. Sociedade Beneficente de Senhoras, São Paulo, SP. Brasil.

Recebido para publicação em 14 de outubro de 1992.

* Do Hospital Sírio Libanês.

Endereço para separatas: José Augusto Báucia. Rua Força Pública, 163. 02012 Sảo Paulo, SP, Brasil. 
BAUCIA, J. A. \& BARBERO-MARCIAL, M. - Extubaçāo precoce em cirurgia cardíaca infantil: procedimentos e resultados em seis anos de experiência. Rev. Bras. Cir. Cardiovasc., 7(3):215-220, 1992.

TABELA 1

DISTRIBUIÇĀO ETÁRIA

\begin{tabular}{|c|c|c|c|}
\hline \multicolumn{2}{|c|}{$I D A D E$} & \multirow{2}{*}{$\frac{N^{9}}{5}$} & \multirow{2}{*}{$\frac{\%}{1,1}$} \\
\hline & $<1 \mathrm{~m}$ & & \\
\hline $1 \mathrm{~m}$ & $<3 m$ & 16 & 3,6 \\
\hline $3 m$ & $<6 \mathrm{~m}$ & 51 & 11,6 \\
\hline $6 m$ & $<12 \mathrm{~m}$ & 135 & 30,6 \\
\hline $1 a$ & $<5 a$ & 153 & 34,7 \\
\hline & $<10 a$ & 70 & 15,9 \\
\hline & $>10 a$ & 11 & 2,5 \\
\hline \multicolumn{2}{|c|}{ TOTAL } & 441 & 100,0 \\
\hline
\end{tabular}

deu-se rotineiramente a extubação ainda em sala cirúrgica, encaminhando-se a criança à UTI com $\mathrm{O}_{2}$ úmido e monitorizaçăo da saturaçāo cutânea de $\mathrm{O}_{2}$, exceto nas crianças com instabilidade hemodinâmica apesar do suporte inotrópico instituído ou persistência de grave hipertensão pulmonar. $O$ procedimento anestésico descrito foi o mais freqüente, com pequenas variaçōes decorrentes de medicamentos em uso, lesão de base, doenças associadas, estado geral e antecedentes anestésico-cirúrgicos.

Nas crianças submetidas a circulação extracorpórea foram utilizadas as técnicas de hipotermia moderada ou profunda com baixo fluxo de perfusão e ocasionalmente a parada circulatória total. O sistema composto por oxigenadores infantís de bolhas ou membranas, circuitos plásticos descartáveis e reservatório de cardiotomia, foi previamente lavado através da circulaçăo de soro fisiológico, seguindo-se adiçăo do perfusato composto por sangue total (ou concentrado globular e plasma fresco) e Ringer Lactato na proporçăo de 2:1. A perfusão iniciou-se com fluxo de $110 \mathrm{ml} /$ $\mathrm{kg} /$ min com indução gradual da hipotermia. Aos $25^{\circ} \mathrm{C}$ de temperatura nasofaríngea, procedeu-se ao pinçamento aórtico e aos $20^{\circ} \mathrm{C}$ o fluxo de perfusão foi reduzido para $60 \mathrm{ml} / \mathrm{kg} / \mathrm{min}$ exceto se o objetivo fosse a parada circulatória total. Durante o período de indução hipotérmica foi administrada Clorpromazina na dosagem de $0,5 \mathrm{mg} / \mathrm{kg}$ e Metilprednisolona na dosagem de $30 \mathrm{mg} / \mathrm{kg}$. O aquecimento foi iniciado previamente ao despinçamento aórtico, aumentando-se o fluxo para 80 e $110 \mathrm{ml} / \mathrm{kg} / \mathrm{min}$ aos $25^{\circ} \mathrm{C}$ e $28^{\circ} \mathrm{C}$, respectivamente. No momento do despinçamento aórtico o fluxo foi reduzido à metade, durante aproximadamente 2 minutos. Ao atingir-se a temperatura de $36,5^{\circ} \mathrm{C}$, mantido por $3 \mathrm{~min}$, e após a recuperação dos batimentos cardíacos, a circulação extracorpórea foi interrompida, com a reduçāo gradual do fluxo de perfusão, de acordo com a capacidade de manutenção do débito cardíaco pelo paciente, desprezando-se no circuito o perfusato excedente e procedendo-se à reposição de volume com sangue fresco, de acordo com a pressāo venosa central e o hematócrito do paciente.

A proteção miocárdica foi realizada através da hipotermia de indução interna a $16^{\circ} \mathrm{C}$ ou $20^{\circ} \mathrm{C}$, da infusão de solução cardioplégica tipo $\mathrm{S}$. Thomas I a $4^{\circ} \mathrm{C}$ em raiz de aorta, na dose inicial de $20 \mathrm{ml} / \mathrm{kg}$, seguindo-se novas infusōes a cada $20 \mathrm{~min}$ ou $30 \mathrm{~min}$ na dose de $10 \mathrm{ml} / \mathrm{kg}$ e da hipotermia tópica intermitente com soro fisiológico a $4^{\circ} \mathrm{C}$ no saco pericárdico.

$\mathrm{Na}$ UTI a criança passou a receber assistência contínua da enfermagem e fisioterapia, cujo programa de atendimento foi estabelecido de acordo com a idade, peso, lesão, antecedentes pessoais, ocorrências no intra-operatório e avaliaçăo conjunta com os médicos intensivista, anestesista e cirurgião. O multiprofissionalismo no período de pós-operatório imediato esteve sempre presente, acentuando a importância do trabalho em equipe de forma coesa e ordenada, com o intuito de qualificar a assistência, reduzir a permanência da criança e conseqüentemente reduzir os custos. Tendo em vista as peculiaridades e as necessidades psicológicas, a criança foi acompanhada por funcionários de enfermagem fixos em cada plantão até a alta. A presença da mãe ou

TABELA 2

PROCEDIMENTOS CIRÚRGICOS/\% EXTUBAÇĀO

\begin{tabular}{lrr}
\hline PROCEDIMENTO & $N^{9}$ & $\%$ \\
\hline CIV* & 72 & 85 \\
T. Fallot & 61 & 82 \\
Anast. S-P & 50 & 86 \\
CIA & 49 & 98 \\
AVC & 43 & 81 \\
CoAo & 32 & 94 \\
TGA & 27 & 59 \\
PCA & 22 & 95 \\
EP & 12 & 83 \\
ESAo & 12 & 92 \\
DATUP & 11 & 82 \\
FONTAN & 10 & 100 \\
DVSVD & 9 & 78 \\
PITVM & 9 & 56 \\
CERCL. TP & 7 & 100 \\
RASTELLI & 5 & 0 \\
Co AN. & 5 & 60 \\
Outros & 17 & 60 \\
\hline
\end{tabular}

CIV: Comunicação Interventricular; T. FALLOT: Tetralogia de Fallot; ANAST. S-P: Anastomose Sistêmico-Pulmonar; CIA: Comunicaçāo Inter-atrial; AVC: Canal Atrioventricular; CoAo: Coarctaçāo de Aorta; TGA: Jransposiçāo das Grandes Artérias; PCA: Persistência do Canal Arterial; EP: Estenose Pulmonar; ESAo: Estenose Sub-Aórtica; DATVP: Drenagem Anômala Total de Veias Pulmonares; DVSVD: Dupla Via de Saída do Ventrículo Direito; P/TVM: Plastia/Troca de Valva Mitral; CERCL. TP: Cerclagem do Tronco Pulmonar; Co AN.: Coronária Anômala; *: $\mathbf{5 0 \%} \mathrm{com}$ anomalias associadas. 
BAUCIA, J. A. \& BARBERO-MARCIAL, M. - Extubaçäo precoce em cirurgia cardíaca infantil: procedimentos e resultados em seis anos de experiência. Rev. Bras. Cir. Cardiovasc., 7(3):215-220, 1992.

outro familiar de melhor relacionamento foi permitida visando suavizar a experiência da criança na UTI, atendendo suas necessidades emocionais e preparando a família para os cuidados a serem tomados após a alta. O tempo médio de permanência em UTI foi de 36 horas, procurando-se, em cardiopatias mais simples como comunicaçōes intracavitárias ou persistência de canal arterial isolados, anastomoses sistêmico-pulmonar, forma parcial, eventualmente total, do canal atrioventricular e muitos dos portadores de tetralogia de Fallot, reduzir a permanência em menos de 24 horas. Otempo médio total de internaçāo foi de sete dias, exceto nos submetidos a anastomose sistêmico-pulmonar, que foi de cinco dias.

\section{RESULTADOS}

Dos 441 pacientes operados, $372(84 \%)$ foram extubados na sala de operaçōes ao término da correção, com base em critérios clínicos, saturação cutânea de $\mathrm{O}_{2}$ e $\mathrm{pO}_{2}$ no ar expirado. Destes, apenas $2(0,5 \%)$ apresentaram insuficiência respiratória necessitando de reentubação nas primeiras horas de pósoperatório.

A medição dos gases arteriais não demonstrou tendência a retenção de $\mathrm{CO}_{2}$ e valores de $\mathrm{pCO}_{2}$ discretamente abaixo do normal em ar ambiente (provavelmente secundário ao edema intersticial pulmonar pós-CEC), foram facilmente controlados com a oferta de ambiente rico em $\mathrm{O}_{2}$.

As complicaçōes pós-operatórias não se correlacionaram com a extubaçāo precoce. As principais foram a instabilidade hemodinâmica (baixo débito cardíaco) em $88(20 \%)$ pacientes, o BAVT transitório em $9(2 \%)$ e o sangramento aumentado em $4(1 \%)$ pacientes.

O índice global de infecção foi de $6,7 \%$, incluindo - de infecção presente no pré-operatório, e o de infecção cirúrgica de $1,2 \%$. Predominou a infecção pulmonar, sendo um óbito relacionado à sepsis secundária a processo pneumônico. Todos os pacientes com infecção pulmonar foram submetidos a ventilaçāo mecânica prolongada.

A mortalidade hospitalar foi de $15(3,4 \%)$ pacientes cujas causas estão relacionadas na Tabela 3.

\section{COMENTÁRIOS}

A cirurgia cardíaca pediátrica possui um universo próprio onde a integração entre os dados clínicos, os recentes avanços tecnológicos e o desenvolvimento de novas técnicas cirúrgicas somam-se, buscando resultados mais efetivos com diminuição da agressão à criança.

Nesse contexto, com a melhoria das técnicas cirúrgicas e anestésicas e especialmente da tecnologia em circulação extracorpórea, a necessidade de ventilação mecânica prolongada no pós-operatório tornase cada vez menos necessária e relatos de bons resultados com a extubação precoce em crianças submetidas a cirurgia cardíaca podem ser observados nos últimos anos. Assim, PRAKASH et alli ${ }^{7}$ relataram que de 142 adultos, $87 \%$ foram extubados

TABELA 3

MORTALIDADE HOSPITALAR

\begin{tabular}{lcll}
\hline CARDIOPATIA & IDADE & PROCEDIMENTO & CAUSAS \\
\hline TAC & $4 \mathrm{~m}$ & RASTELLI & SBD \\
AT & $3 \mathrm{a}$ & FONTAN & SBD \\
CIV & $9 \mathrm{~m}$ & C. TOTAL & IP \\
COMPLEXA & $2 \mathrm{a}$ & ANAST. S-P & SBD \\
CIV & $5 \mathrm{~m}$ & C. TOTAL & SBD \\
CIV+PCA & $10 \mathrm{~m}$ & C. TOTAL & SBD \\
CIV MÚLTIPLAS & $2 \mathrm{~m}$ & C. TOTAL & SBD \\
CIV + PCA & $10 \mathrm{~m}$ & C. TOTAL & SBD \\
AVC & $1 \mathrm{a}$ & TVM & SBD \\
CIV + IAAO & $7 \mathrm{~m}$ & C. TOTAL & SBD+SH \\
COMPLEXA & $6 \mathrm{a}$ & C. TOTAL & SBD \\
IM & $3 \mathrm{a}$ & TVM & SBD \\
COMPLEXA & $1 \mathrm{a}$ & ANAST. S-P & SÚBITO \\
AP & $7 \mathrm{~d}$ & ANAST. S-P & TAPE \\
TGA & $3 \mathrm{~m}$ & C. ANATOMMICA & IAM \\
\hline
\end{tabular}

TAC: Tronco Arterioso Comum; SBD: Síndrome de Baixo Débito; AT: Atresia Tricúspide; IP: Infecção Pulmonar; TVM: Troca de Valva Mitral; IAAo: Interrupçāo do Arco Aórtico; SH: Síndrome Hipertérmica; IM: Insuficiência Mitral; TAPE: Trombose de Artéria Pulmonar Esquerda; IAM: Infarto Agudo do Miocárdio. 
BAUCIA, J. A. \& BARBERO-MARCIAL, M. - Extubaçāo precoce em cirurgia cardíaca infantil: procedimentos e resultados em seis anos de experiência. Rev. Bras. Cir. Cardiovasc., 7(3):215-220, 1992.

em sala cirúrgica ou após 3 horas de pós-operatório, permanecendo menor número de dias em UTI sem aumento na morbidade pulmonar. LICHTENTHAL et alii ${ }^{6}$ relataram 100 adultos, $40 \%$ com extubação em até 90 minutos de pós-operatório sem necessidade de reentubaçăo. BARASH et alii ${ }^{2} \mathrm{em}$ revisão da literatura concluíram que a ventilação mecânica prolongada tem sido cada vez menos empregada em pacientes pediátricos e apresentaram um protocolo para extubação precoce envolvendo vários critérios como testes de funçāo pulmonar. HEARD et alii ${ }^{5}$ relataram 220 pacientes submetidos a correção de cardiopatias congênitas, $67 \% \mathrm{com}$ extubaçāo em sala cirúrgica ou após 6 horas de pós-operatório, incluindo alguns com lesōes mais complexas; utilizaram simples critérios clínicos com redução das complicaçōes pulmonares potenciais e dos custos hospitalares. QUASHA et alii ${ }^{8}$, em estudo comparativo de 2 grupos de pacientes submetidos a revascularizaçāo do miocárdio quanto a extubaçăo precoce $(2 \pm 2$ horas) ou tardia ( $18 \pm 3$ horas), obtiveram sucesso em $89 \%$ dos casos de extubaçáo precoce realizada e concluíram que o método possibilitou a administração de menor quantidade de sedativos no pós-operatório imediato, diminuindo consideravelmente a morbidade cardiopulmonar.

A conduta anestésica empregada em nossos pacientes visa à extubação precoce ${ }^{4}$. Em nossa casuística $372(84 \%)$ pacientes foram extubados em sala cirúrgica, representando a maior série da litera- tura. Devido ao suporte fisioterápico intensivo, cujos objetivos são a preservação das condiçōes de ventilação pulmonar adequada através de manobras que mantenham livre as vias aéreas, mobilidade e expansibilidade torácica e correção de postura antálgica, apenas $2(0,5 \%)$ pacientes necessitaram reentubação por depressão da função respiratória. Considerando os pacientes operados a cada ano, verificamos que houve um aumento percentual progressivo dos casos de extubaçāo precoce, incluindo procedimentos mais complexos e não presentes em outras casuísticas (correção atrial e anatômica da transposiçāo das grandes artérias, tetralogia de Fallot e forma total do canal atrioventricular), demonstrando que este procedimento vem sendo consagrado pelos resultados obtidos (Gráfico 1).

Vários sāo os efeitos deletérios da circulação extracorpórea no subsistema pulmonar levando a insuficiência respiratória. RATLIFF et alii ${ }^{10} \mathrm{e} C O N N E L L$ et alii ${ }^{3}$ reportaram em biopsia pulmonar pós-CEC, edema intersticial, hemorragia perivasculer e atelectasias miliares. Componente indispensável na rotina da cirurgia cardiaca, o sangue pode ser um perigoso recurso terapêutico. RABELO et alii ${ }^{\circ}$, pesquisando as causas de insuficiência respiratória pós-CEC, identificaram alteraçōes pulmonares à microscopia eletrônica que relacionaram ao uso, de sangue estocado como perfusato. $O$ fato de nossos pacientes submetidos a extubaçāo precoce nāo apresentarem insuficiência respiratória, que corresponde a manifestação

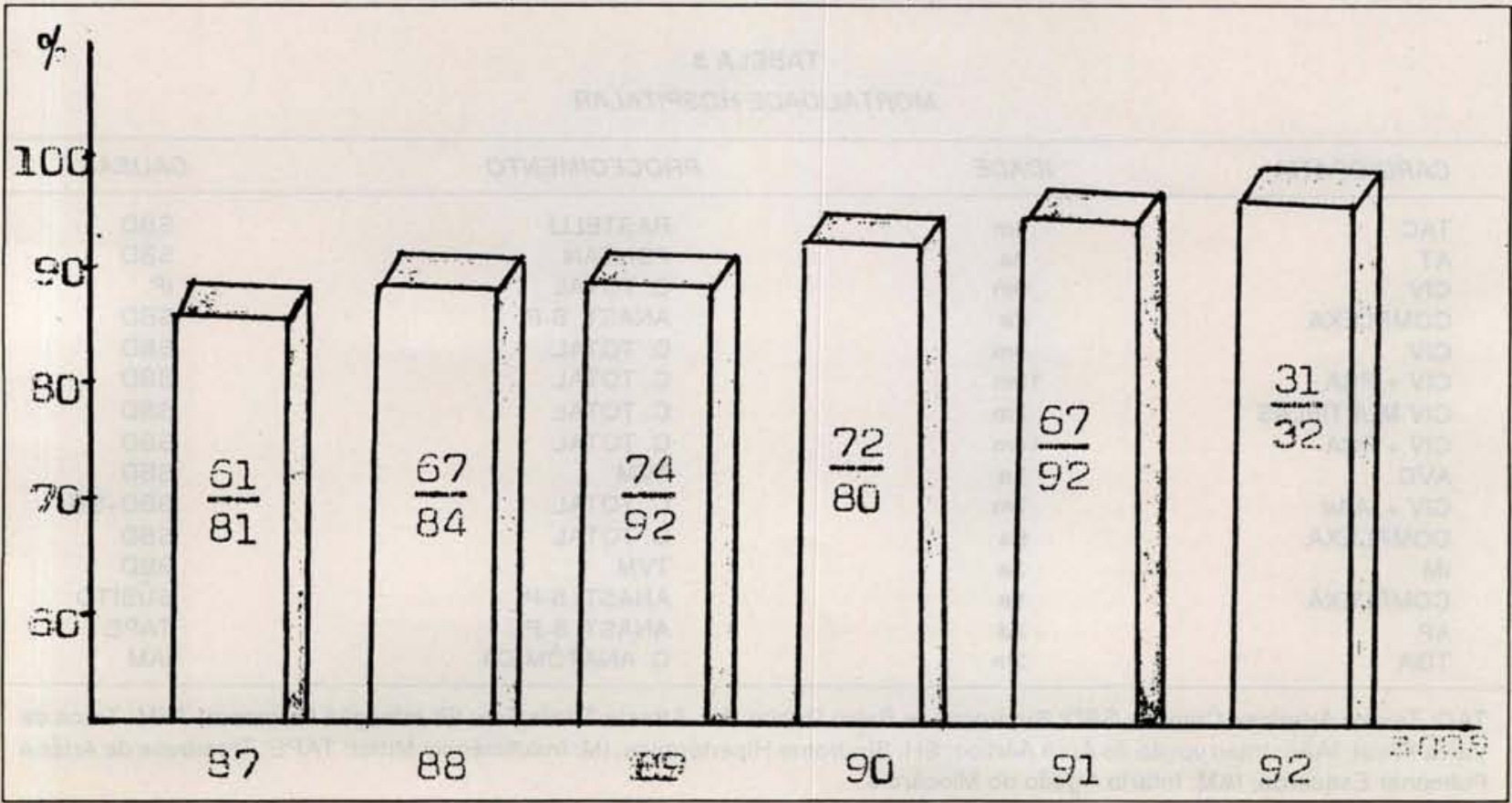


BAUCIA, J. A. \& BARBERO-MARCIAL, M. - Extubação precoce em cirurgia cardíaca infantil: procedimentos e resultados em seis anos de experiência. Rev. Bras. Cir. Cardiovasc., 7(3):215-220, 1992.

clínica das alteraçōes pulmonares referidas, sugere que o procedimento de estocagem pode estar envolvido na sua fisiopatologia.

A incidência de infecçāo em crianças submetidas a cirurgia cardíaca em nosso Serviço foi estudada por AYROSA GALVĀO et alii ${ }^{1}$. Entre os que apresentaram manifestaçōes infecciosas, $84 \%$ tinham menos de 1 ano de idade (compreendendo $12,2 \%$ das crianças nesta faixa etária) e 2,1\% em crianças acima de 1 ano de idade. As manifestaçōes encontradas foram respiratórias em $66 \%$, urinárias em $16 \%$ e cutâneas em $16 \%$, sendo que todas as crianças que apresentaram infecção respiratória foram submetidas a ventilação mecânica prolongada.
Concluímos que a grande maioria das crianças portadoras de cardiopatias congênitas submetida a intervençōes cirúrgicas, incluindo lesōes complexas e com hipertensāo pulmonar pré-operatória, pode ser extubada com segurança imediatamente após o procedimento, com base em critérios clínicos, saturação cutânea de $\mathrm{O}_{2}$ e $\mathrm{pCO}_{2}$ no ar expirado, isso diminui as complicaçōes pulmonares secundárias a ventilaçāo mecânica (atelectasias secundárias a obstrução mucosa de bronquíolos, traumas de laringe e traquéia, infecção e pneumotórax); diminuindo o período de permanência em UTI e de internaçāo, atende às necessidades emocionais da criança, atenua a ansiedade dos familiares, diminui a possibilidade de infecção hospitalar e reduz significativamente os custos.

\section{RBCCV/44205-182}

BAUCIA, J. A. \& BARBERO-MARCIAL, M. - Early extubation in pediatric cardiac surgery: proceedings and results in six years experience. Rev. Bras. Cir. Cardiovasc., 7(3):215-220, 1992.

ABSTRACT: Of 441 pediatric patients recovering from surgical repair of congenital heart disease, 372 $(84 \%)$ were extubated in the operating room immediately after the procedure, using a clinical criteria, cutaneous $\mathrm{pO}_{2}$ saturation and $\mathrm{pCO}_{2}$ in exchanged air. Postoperative complications were not correlated with the procedure, and less pulmonary complications were observed. We concluded that most of pediatric patients, including many of those with complex lesions and preoperative pulmonary hipertension, can safely be extubated early cardiac surgery with minimization of pulmonary complications secondary to mechanical ventilation as well as reduced length of $\mathrm{ICU}$ and hospitalary stay, minimizing children and parents stress and hospitalary cost.

DESCRIPTORS: pediatric cardiac surgery, early extubation.

\section{REFERÊNCIAS BIBLIOGRÁFICAS}

1 AYROSA-GALVÄO, P. C.; GONZAGA, R. L.; MITRE, N. - Heart surgery in children: postoperative in a general intensive care unit. Rev. Bras. Terap. Intens., 3 (Supl. I): 122, 1991.

BARASH, P. G.; LESCOVICH, F.; KATZ, J. D.; TALNER, N. S.; STANSEL Jr., H. C. - Early extubation following pediatric cardiothoracic operation: a viable alternative. Ann. Thorac. Surg., 29: 228-233, 1980.

CONNELL, R. S.; PAGE, V. S.; BARTLEY, T. D.; BIGELOW, J. C.; WEEB, M. C. - The effect on pulmonary ultrastructure of Dacron-Wool filtration during cardiopulmonary bypass. Ann. Thorac. Surg., 15: 217 229, 1973.

FREIRE SOBRINHO, A.; BAUCIA, J. A.; TRANQUI-
TELLE, A. M.; NAKAGAWA, N. K.; BARBERO-MARCIAL, M. - Cirurgia cardíaca infantil em hospital geral: procedimentos e resultados em 5 anos de experiência. Arq. Bras. Cardiol, (No prelo).

5 HEARD, G. G.; LAMBERT Jr., J. J.; PARK, S. M.; WALD. MAN, J. D.; WALDMAN, J. - Early extubation after surgical repairy of congenital heart disease. Crit. Care Med., 13: 830-836, 1986.

6 LICHTENTHAL, P. R.; WADE, L. D.; NIEMYSKI, P. R. Respiratory management after cardiac surgery with inhalation-anesthesia. Crit. Care Med., 11: 603609, 1983.

7 PRAKASH, O.; JOHNSON, B.; MEIJI, S.; BOS, E.; HUGENHOLTZ, P. G.; NAUTA, J.; HEKMAN, W. - Criteria for early extubation after intracardiac surgery in adults. Anesth. Analg., 59: 703-708, 1977. 
BAUCIA, J. A. \& BARBERO-MARCIAL, M. - Extubação precoce em cirurgia cardíaca infantil: procedimentos e resultados em seis anos de experiência. Rev. Bras. Cir. Cardiovasc., 7(3):215-220, 1992.

8 QUASHA, A. C.; LEOBER, N.; FEELEY, T. W.; ULLYOT, D. J.; ROIZEN, M. F. Postoperative respiratory care: a controlled trial of early and late extubation following coronary-artery bypass grafting. Anesthesiology, 52: 135-141, 1980.

9 RABELO, R. C.; OLIVEIRA, S. A.; TANAKA, H.; WEIGL, D. R.; VERGINELLI, G.; ZERBINI, E. J. - The influence of the nature of the prime on postperfusion pulmonary changes. J. Thorac. Cardiovasc. Surg., 66: 782, 1973.

10 RATLIFF, N. B.; YOUNG, W. G.; HACKEL, D. B.; MIKAT, E.; WILSON, W. - Pulmonary injury secondary to extracorporeal circulation: an ultrastructural study. $J$. Thorac. Cardiovasc. Surg., 65: 425-432, 1973. 